\title{
Téoros
}

Revue de recherche en tourisme

\section{Pour prolonger et élargir la réflexion...}

Trois points de vue sur la place de l'État dans l'économie de marché

Volume 3, numéro 2, juillet 1984

L'intervention étatique dans le domaine du tourisme : premiers

coups de sonde

URI : https://id.erudit.org/iderudit/1080805ar

DOI : https://doi.org/10.7202/1080805ar

Aller au sommaire du numéro

Éditeur(s)

Université du Québec à Montréal

ISSN

0712-8657 (imprimé)

1923-2705 (numérique)

Découvrir la revue

Citer ce document

(1984). Pour prolonger et élargir la réflexion... Trois points de vue sur la place de l'État dans l'économie de marché. Téoros, 3(2), 31-32.

https://doi.org/10.7202/1080805ar d'utilisation que vous pouvez consulter en ligne.

https://apropos.erudit.org/fr/usagers/politique-dutilisation/ 


\section{Pour prolonger et élargir la réflexion...}

\section{La présence de l'État en évolution}

Ce premier point de vue à caractère descriptif est extrait de Batir le Québec. énoncé de politique économique du gouvernement québecois ${ }^{i 11}$.

\section{L'État - agent économique}

L'évolution de la présence de l'État est une des principales caractéristiques des sociétés modernes. Le poids relatif du secteur public ne cesse de progresser dans tous les pays industrialises, surtout depuis la fin de la deuxième guerre mondiale. II $n$ 'existe pratiquement plus aucun champ d'activités oủ les gouvernements ne sont pas appelés à agir. Même dans les sociétés les plus libérales, il est de plus en plus fréquent et admis que certaines interventions publiques viennent compléter le rôle traditionnellement réservé au secteur privé. Ainsi, le gouvernement américain a annoncé récemment la création d'une société dotée d'un fonds d'investissement de près de 90 \$ milliards pour encourager, dans le domaine énergétique, la production de carburant de synthèse.

On reconnaît ordinairement à l'État moderne six grandes fonctions : la protection, la réglementation, la production de biens et services, la compensation ou la redistribution des revenus, la stabilisation et la stimulation de la croissance économique. Ces fonctions ne s'exercent pas indépendamment les unes des autres mais sont interreliées, si bien que les décisions doivent tenir compte des effets qui se répercutent simultanément sur la répartition des revenus, sur l'affectation des ressources, sur la stabilité et la croissance de l'activité économique. L'action gouvernementale a presque toujours une influence directe ou indirecte sur le mécanisme des prix, le fonctionnement de l'économie du marché, la répartition des facteurs de production et, somme toute, sur l'évolution du secteur privé (...).

\section{L'État - agent actif de développement}

Les programmes sociaux, éducatifs et culturels ont connu dans tous les pays industrialisés un rythme d'expansion exceptionnel, surtout depuis le début des années soixante, et il est indéniable qu'au Canada et au Québec l'augmentation de la production de biens et de services publics a été accompagnée d'une très forte hausse des effectifs des secteurs public et parapublic et d'un gonflement de la masse salariale beaucoup plus rapide que dans le secteur privé. Cette remarque ne remet pas en cause le bien-fondé des services publics qui, dans la très grande majorité des cas, répondent à des besoins essentiels mais cette situation suscite de très nombreuses questions quant au contrôle des coûts et à la uvéritén des prix exigés des bénéficiaires (...).

11) Gouvernement du Québec. Bâti le Québec énoncé de politique économique, Ouébec, 1979 . pp. 43 et 51.

\section{L'État - producteur de biens et services}

Le gouvernement du Québec est aussi un producteur de biens et services «privésw. II possède près d'une vingtaine d'entreprises financières, commerciales ou industrielles qui bénéficient d'un monopole juridique (I'Hydro-Québec, la Société des alcools) ou qui sont en concurrence avec d'autres entreprises (SGF, SIDBEC, SOQUEM, SOQUIA, etc.l. Seulement quatre d'entre elles ont vu le jour avant 1960. Certaines sociétés d'Ėtat québécoises sont loin d'être marginales et se classent parmi les grandes entreprises du Québec (HydroQuébec, SGF, SIDBEC, Caisse de dépöt et de placementl. Cependant, par rapport à la production nationale, l'ensemble des entreprises publiques québécoises occupent encore aujourd' hui une place relativement faible. Les activités financières, industrielles ou commerciales des sociètés d'État sont, toutes proportions gardées, moins importantes au Québec que dans la plupart des pays industrialisés (...). 


\section{La présence nationaliste}

Un deuxieme point de vue est formule par Claude Forget, ex-ministre liberral et maintenant associe de SECOR inc. et directeur associe de /linstitut C. D. Howe. Claude Forget a fait part de ses réflexions dans le journal Les Affaires dans le cadre d'un article sur les rapports entre les societes d'Etat et /'entreprise privée ${ }^{|2|}$.

D'autres sociétés d'État visent à assurer une aprésences et reflètent le sentiment nationaliste canadien (au niveau des sociétés d'État fédérales) ou québeécois francophone lquant au $x$ sociétés d'Etat du Québecl.

La SGF de la Caisse de dépôt, la Société de développement du Canada; Air Canada et Quebecair; Pétro-Canada et SOQUIP: Canadair et De Havilland semblent appartenir à ce groupe. Ces sociètés constituent des symboles auxquelles Canadiens et Québécois semblent attachés, mais dans la mesure seulement où ces entreprises sont a la hauteur des standards de l'entreprise privée, c'est-ă-dire profitables.

II me semble que ces initiatives gouvernementales dans le monde de la finance ou de l'industrie constituent une réponse à l'anxiété souvent éprouvée face au acontrôle étrangers. Si cela est vrai, i s'agit d'une donnée de base de la scêne québécoise et canadienne et d'une caractéristique permanente que les gouvernements seraient mal avisés de vouloir gommer par un recours massif à la privatisation.

En tant que réponses à la question posée par le niveau élevé de controble étranger de I'industrie canadienne, ces initiatives sont, à tout prendre, plus positives que la réglementation des investissements étrangers à la FIRA. Si, comme il est probable, ce dernier type de réglementation est à plus ou moins long terme condamné a disparaître, l'appui envers les sociétés d'Etat qui assurent une présence dans certains secteurs jugés kimportants: s'accroitera d'autant.

Cependant une aprésences canadienne ou québécoise n'a de valeur symbolique ou, en définitive, d'impact positif qu'à condition de connaitre le succes financier et de se plier aux mêmes règles que les entreprises analogues du secteur privé. II s'agit lá de standards de performance auxquels elles ne peuvent pas toujours satisfaire, mais qu'elles ignorent à leur péril. C'est aussi ce qui peut leur procurer des relations tolérables avec l'entreprise privée (...).

(2) FonGET, Claude, Les sociétés d'Etat ot Fentreprise privde; coexistence pacifique ou hostile? dans Les Affaires, 26 mai $1984_{f}$ pp. 525 .

\section{Plus d'État et plus de marché}

Alain Minc est français. Directeur financier d̀ trente-quatre ans d'une grande entreprise publique francaise, II est aussi l'auteur d'un live qui a fait couler beaucoup d'encre L'Apres-crise est commencée ${ }^{(3)}$ ou il expose ce qu'il appelle le modele uétalo-libertairew. Dans une entrevue dans Le Monde Dimanche ${ }^{(4)}$, d expose son point de vue :

$\alpha$ Dans ce contexte, je crois qu'il faut redécouvrir les vertus du marché, notamment lorsqu'il s'applique à la sphère sociale, car il exerce en général des effets dissolvants sur les oligopoles et les néo-corporatismes. Entendons-nous: le marché ne s'assimile pas forcément à la privatisation du capital ou à une marchandisation exacerbée. II signifie simplement I'introduction d'un minimum de pression concurrentielle. c'est-ä-dire en definitive l'obligation pour des organisations trop stables de s'ajuster et de s'adapter. Le marché limite considerablement les risques de dérive bureaucratique des grandes organisations publiques ou privées : c'est une force de régulation tellement puissante, tellement brutale parfois, qu'on ne peut y échapper longtempss.

\section{Le profit incontournable}

aD'où la nécessité d'une uréhabilitation de gauchen du profit. Celui-ci est une réalité incontoumable en univers concurrentiel, particulièrement lorsque cet univers est international. Pourquoi une réhabilitation de gauche? Parce que je pense, comme Braudel, que le marché est un état de nature de la société et qu'il est un des moyens privilégiés pour une société d'assurer son propre mouvement et son propre changement. Le marché n'est pas conservateur. II est parfois révolutionnaire, et en tout cas réformiste puisqu'il engendre le mouvementw.

qll reste que s'il faut davantage de marché dans l'univers bureaucratique pour en contenir le mouvement naturel qui le porte vers l'entropie, il faut davantage d'État là où le vent de la concurrence internationale souffle largement. II n'y a là rien de très nouveau : dans les périodes de crise durable, l'Ėtat s'est toujours porté au secours du système productif. Davantage d'Etat ne signifie pas un Etat producteur. Cela signifie un État qui aide certaines entreprises à ruser avec le marché en les dotant mieux sur le plan financier que ne l'aurait fait parfois un marché boursier historiquement anémié, en leur permettant de jouer davantage le long terme sans s'affranchir des rigueurs du quotidiens.

A la fois plus d'État lă où le système productif a besoin de soutien, plus de marché pour réguler les énormes machines bureaucratiques et plus d'espaces de liberté dans le jeu social (...).
(3) MiNC, Alain, L'apress-crise est commencete, Paris, Gallimard, 1982.

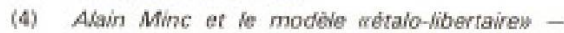
entretien dans Le Monde Dimanche, 10 juillet 1983, p. XI. 